\title{
Déserts alimentaires à Winnipeg (Canada) : une nouvelle méthodologie de mesure d'un concept complexe et controversé
}

\author{
Joyce Slater, Ph. D. (1); Stefan Epp-Koop, M.A. (2); Megan Jakilazek, B. Sc. (3); Chris Green, Ph. D. (3)
}

Cet article a fait l'objet d'une évaluation par les pairs.

Diffuser cet article sur Twitter

\section{Résumé}

Introduction : Les « déserts alimentaires » ont vu le jour dans les 20 dernières années et forment des secteurs préoccupants pour les collectivités, les autorités en santé publique et les chercheurs en raison de leur effet négatif possible sur la qualité de l'alimentation et en raison de leurs conséquences sur la santé. Ce sont des espaces résidentiels, habituellement en milieu urbain, où les résidents à faible revenu n'ont que peu ou pas accès à des établissements de vente au détail d'aliments qui offrent suffisamment de variété à un prix abordable. La recherche sur les déserts alimentaires présente des défis méthodologiques, notamment la façon de repérer et de classer les magasins d'alimentation au détail, la définition de la population à faible revenu ainsi que les paramètres concernant le transport et la proximité. De plus, les méthodes complexes qui sont souvent employées dans la recherche sur les déserts alimentaires peuvent être difficiles à reproduire et à communiquer aux principaux intervenants. Pour surmonter ces difficultés, nous avons voulu montrer qu'on pouvait concevoir une méthode simple et reproductible pour repérer les déserts alimentaires, à l'aide de données facilement accessibles en contexte canadien.

Méthodologie : Cette étude a été menée à Winnipeg (Canada) en 2014. Les établissements de vente au détail des aliments ont été trouvés à l'aide des Pages Jaunes et vérifiés par des diététistes en santé publique. Nous avons créé deux scénarios sur les déserts alimentaires en fonction de l'emplacement de la population à quintile de revenu le plus faible : a) celle qui habitait à $500 \mathrm{~m}$ ou plus d'une épicerie appartenant à une chaîne nationale et b) celle qui habitait à $500 \mathrm{~m}$ ou plus d'une épicerie appartenant à une chaîne nationale ou d'une épicerie à service complet.

Résultats : En fonction du scénario utilisé, 64574 ou 104335 résidents à faible revenu vivaient dans un désert alimentaire.

Conclusion : Les déserts alimentaires touchent une proportion importante de la population de Winnipeg et, même s'ils sont concentrés en centre-ville, ils sont présents également en banlieue. La méthodologie employée pour repérer les déserts alimentaires est accessible, claire et reproductible. Elle est utilisable pour exercer une surveillance périodique à faible coût, ainsi que pour favoriser un engagement significatif de la part des collectivités, des magasins de détail et des responsables des politiques.

Mots-clés : désert alimentaire, SIG, sécurité alimentaire, Canada

\section{Introduction}

Les " déserts alimentaires » ont vu le jour dans les 20 dernières années : ce sont des espaces géographiques résidentiels, habituellement en milieu urbain, où les résidents à faible revenu n'ont que peu ou pas accès à des établissements de vente au détail d'aliments qui offrent suffisamment de variété à un prix abordable ${ }^{1}$. Ce sont des secteurs préoccupants pour les collectivités, les autorités de la santé publique et les chercheurs en raison de leur effet négatif possible sur la qualité de l'alimentation et

\section{Points saillants}

- Même si les "déserts alimentaires" sont un concept controversé, les évaluer et les décrire est utile pour stimuler la discussion sur la façon de lutter contre l'insécurité alimentaire et les inégalités.

- En 2014, 9 \% de la population de la Région sanitaire de Winnipeg vivait dans un désert alimentaire urbain, c'est-à-dire une région à faible revenu située à $500 \mathrm{~m}$ ou plus d'une épicerie appartenant à une chaîne nationale ou à une épicerie à service complet.

- Si la majorité des quartiers qualifiés de déserts alimentaires étaient situés dans le centre-ville de Winnipeg, certains ont été repérés en banlieue éloignée.

- Des méthodes simples et économiques tenant compte du revenu, de l'emplacement des magasins d'alimentation, des effectifs de population et des vérifications factuelles permettent de déterminer de façon raisonnable l'emplacement et la superficie des déserts alimentaires urbains. Ces méthodes sont également utilisables pour exercer une surveillance continue.

- Le fait de ne pas tenir compte des épiceries locales à service complet lors du repérage des déserts alimentaires peut conduire à une surestimation de leur superficie.

la quantité d'aliments offerts. Les résidents des déserts alimentaires peuvent en effet dépendre des petits magasins de détail, comme les dépanneurs, qui offrent peu de choix et qui vendent généralement la 
plupart des produits alimentaires à des prix plus élevés que les autres magasins. La situation de ces résidents est aggravée du fait qu'ils n’ont pas nécessairement les ressources financières pour se procurer une voiture ou pour trouver un autre moyen de transport appropriée,3. L'absence d'épiceries à service complet offrant des produits à un prix raisonnable peut donc aussi accroître les inégalités, les résidents risquant davantage de mal s'alimenter, ce qui a une incidence négative sur leur santé à long terme $e^{4-6}$.

Cependant, il est important de noter qu'il n'existe aucune définition commune de " désert alimentaire ", la littérature offrant différentes interprétations, principalement basées sur les méthodologies utilisées, qui varient grandement ${ }^{7,8}$. Certains auteurs remettent en question l'utilité du concept de désert alimentaire : ils soutiennent que ce concept masque un enjeu prioritaire, un revenu insuffisant, et qu'il importe peu qu'il y ait ou non une épicerie à service complet à proximité ${ }^{9}$. D'autres ont tenté de tenir compte de cette dimension en utilisant le concept de "mirage alimentaire ", c'est-à-dire un quartier abritant des magasins à service complet, mais inaccessibles aux résidents à faible revenu en raison d'un faible pouvoir d'achat ${ }^{10,11}$. On peut donc conclure que le désert alimentaire est un concept controversé, de nouvelles interprétations et méthodes étant continuellement proposées.

À ce jour, la présence et les caractéristiques des déserts alimentaires ont principalement été étudiées en milieu urbain en Australie, au Royaume-Uni, aux États-Unis et au Canada $^{12-16}$. Les résultats de ces travaux de recherche sont ambigus. Dans le cadre d'une revue de la littérature, Beaulac et coll. ${ }^{17}$ ont constaté qu'il existait des disparités évidentes quant à l'accès aux aliments en fonction du revenu et de l'origine ethnique dans un grand nombre de municipalités américaines, mais pas dans toutes. Des déserts alimentaires ont été répertoriés dans des villes d'Australie ${ }^{12}$ et du Royaume$\mathrm{Uni}^{18}$ et, au Royaume-Uni, l'ouverture d'une épicerie à service complet n'a pas modifié l'alimentation des résidents ${ }^{19}$. Des travaux de recherche effectués au Canada indiquent que certaines villes abritent des déserts alimentaires, dont London (Ontario), où la population à faible revenu qui vit au centre-ville est la plus défavorisée quant à l'accès aux supermarchés ${ }^{20}$, et Gatineau (Québec), où 7,5 \% de la population dispose de ressources financières limitées et a difficilement accès à des aliments sains ${ }^{21}$. Cependant, un faible revenu n'est pas toujours associé à un accès réduit aux aliments. Ainsi, on trouve des déserts alimentaires importants dans certains quartiers à faible revenu d'Edmonton et de Saskatoon, mais d'autres sont situés dans des secteurs qui comportent beaucoup d'épiceries ${ }^{22,23}$. Des tendances similaires ont été observées dans des centres urbains du sud de l'Ontario ${ }^{15,24}$. Ces observations ont été confirmées dans une étude récente de Minaker et coll. sur l'environnement alimentaire canadien ${ }^{25}$. Les auteurs ont conclu que l'association entre le faible revenu et les difficultés d'accès aux aliments était plus marquée aux États-Unis qu'au Canada, où les résidents de nombreux quartiers urbains défavorisés ont accès à des aliments sains aussi facilement que dans les quartiers aisés, voire plus facilement.

$\mathrm{Au}$ sein d'une même zone urbaine, des conclusions différentes ont pu été tirées sur la présence et les caractéristiques des déserts alimentaires. C'est par exemple le cas de trois études réalisées dans la ville de Montréal (Québec). Apparicio et ses collègues ont constaté que l'accessibilité géographique aux aliments sains n'était pas difficile, et ils en ont donc conclu que les déserts alimentaires ne constituaient pas un problème à Montréal ${ }^{26}$. De leur côté, Bertrand et ses collègues ont conclu qu'une proportion importante de la population sans véhicule avait difficilement accès aux fruits et légumes ${ }^{27}$. Bien qu'ils n'aient pas utilisé le terme " déserts alimentaires ", Páez et ses collègues ont quant à eux constaté des différences d'accès aux aliments sains chez les Montréalais à faible revenu en fonction de l'endroit où ils habitaient ${ }^{2}$. Ces résultats variés et souvent contradictoires découlent des différentes méthodologies utilisées pour repérer et définir les déserts alimentaires, en particulier les méthodes d'analyse spatiale employées, la prise en compte ou non de l'accès au transport, le type et la variété des magasins de détail analysés, le degré de détail et la complexité des méthodes utilisées et enfin la validation ou non des données et des résultats par des méthodes qualitatives appropriées.

Les méthodes complexes et les données détaillées utilisées dans de nombreuses études sur les déserts alimentaires, qui nécessitent souvent une importante collecte de données brutes, constituent un problème de taille, car ces études peuvent être difficiles et coûteuses à reproduire.
C'est le cas par exemple de celle de Luan²4, qui a évalué de façon très détaillée l'environnement alimentaire de Waterloo, mais en utilisant un certain nombre de méthodes et de variables complexes (p. ex. l'accès relatif à des aliments sains, les tendances spatiotemporelles et la modélisation hiérarchique). De plus, l'utilisation de méthodes complexes peut nuire à l'application efficace des connaissances en dehors du milieu universitaire, car elles sont difficiles à expliquer et peuvent de ce fait offrir moins de crédibilité pour la population et les responsables des politiques. En outre, comme le paysage alimentaire contemporain est dynamique (il arrive régulièrement que des magasins de détail disparaissent des collectivités ou s'y installent), il est essentiel de pouvoir mettre à jour régulièrement les analyses concernant les déserts alimentaires, afin qu'elles demeurent d'actualité et pertinentes. Les méthodes complexes et exigeantes sur le plan des ressources sont susceptibles de rendre cette tâche difficile à accomplir dans un temps acceptable.

Malgré la controverse autour des déserts alimentaires et l'absence de consensus sur les méthodes appropriées pour les évaluer ou les décrire, le concept de désert alimentaire est devenu important, car il facilite la discussion, les débats et les négociations au sein des collectivités (ou entre les collectivités et les responsables des politiques) sur la façon de lutter contre l'insécurité alimentaire dans le contexte du paysage alimentaire contemporain ${ }^{28}$. Bien que l'on ne s'entende pas sur ce qu'est un " désert alimentaire ", le terme est maintenant reconnu dans les domaines du développement communautaire et de la santé publique. Ce concept est utile pour attirer l'attention sur les problèmes liés à l'environnement alimentaire et à l'insécurité alimentaire. L'objectif de cette étude a été dans ce contexte de démontrer qu'on pouvait concevoir une méthode reproductible pour repérer les déserts alimentaires, et ce, en utilisant un minimum de ressources et des données facilement accessibles dans le contexte canadien de la santé publique. Étant donné que la vaste majorité des services de santé publique du Canada ne peuvent recourir à des méthodes onéreuses, l'approche proposée offre un modèle pragmatique permettant la mise en place d'une surveillance du paysage alimentaire en contexte de santé publique. 


\section{Méthodologie}

L'étude a été menée dans la Région sanitaire de Winnipeg, constituée de la ville de Winnipeg et de deux municipalités rurales adjacentes, dans la province canadienne du Manitoba (au centre du Canada). La Région sanitaire de Winnipeg comptait 736000 habitants en 2014.

Nous avons utilisé trois sources de données (décrites ci-dessous) pour créer deux scénarios sur les déserts alimentaires (tableau 1), en fonction de la proximité de deux catégories de magasins d'alimentation au détail : les épiceries appartenant à une chaîne nationale et les épiceries à service complet. Les épiceries appartenant à une chaîne nationale ont été définies comme de grandes épiceries, dont l'enseigne est présente au Manitoba et dans d'autres provinces et qui offrent un service complet. Les épiceries à service complet ont été définies comme de grandes épiceries mais locales (n'appartenant pas à une chaîne nationale) proposant une bonne sélection de fruits et légumes frais (c.-à-d. davantage que des pommes de terre, des oignons et des bananes, et avec des produits non préemballés), de la viande fraîche et des produits laitiers à des prix raisonnables (c.à-d. des prix se rapprochant de ceux des épiceries appartenant à une chaîne nationale). Ces critères ont été analysés par des diététistes en santé publique de la région participant à l'étude et connaissant bien les épiceries locales et leurs caractéristiques ainsi que le prix des aliments. Les diététistes ont reçu une liste des épiceries sélectionnées, ont déterminé si elles avaient été bien classées et ont également recensé les épiceries qui ne figuraient pas sur la liste et celles qui avaient fermé.

Nous avons en premier lieu créé une base de données contenant toutes les épiceries appartenant à une chaîne nationale et toutes les épiceries à service complet exerçant leurs activités dans la Région sanitaire de Winnipeg à ce moment-là. Les données initiales ont été tirées des Pages Jaunes de l'annuaire téléphonique de Winnipeg et des environs (version imprimée). Les diététistes et facilitateurs communautaires de l'Office régional de la santé de Winnipeg ont ensuite vérifié et affiné cette base de données initiale pour s'assurer qu'elle reflétait bien la situation locale.

Nous avons en deuxième lieu utilisé les données du recensement canadien de 2011 à l'échelle de l'aire de diffusion pour répartir

TABLEAU 1

Scénarios utilisés pour repérer les déserts alimentaires à Winnipeg (Manitoba), Canada

îlot de diffusion dans le quintile de revenu le plus faible, et

Scénario 1 - Désert alimentaire

Centroïde d'un îlot de diffusion à $500 \mathrm{~m}$ ou plus d'une épicerie appartenant à une chaîne nationale.

îlot de diffusion dans le quintile de revenu le plus faible, et

Scénario 2 - Désert alimentaire Centroïde d'un îlot de diffusion à $500 \mathrm{~m}$ ou plus d'une épicerie appartenant à une chaîne nationale OU d'une épicerie à service complet.

les 5500 îlots de diffusion de la Région sanitaire de Winnipeg en quintiles, selon le revenu moyen du ménage et selon les seuils de revenu établis par le Centre manitobain des politiques en matière de santé29. Chaque quintile de revenu renferme environ $20 \%$ de la population de Winnipeg (tableau 2). Dans le cadre de notre étude, le revenu moyen du ménage a servi d'indicateur du pouvoir d'achat de la population concernant les aliments et a également été utilisé comme indicateur substitutif de la possibilité de posséder une voiture. On a attribué aux îlots de diffusion la classification en quintiles de revenu de l'aire de diffusion dont ils faisaient partie. L'aire de diffusion est la plus petite unité géographique pour laquelle Statistique Canada diffuse des données de recensement détaillées, et il en existe 1150 dans la Région sanitaire de Winnipeg. Un îlot de diffusion est plus petit qu'une aire de diffusion (il y a environ cinq îlots de diffusion par aire de diffusion), mais aucune donnée de recensement détaillée n'est disponible à cette échelle.

Nous avons en troisième lieu extrait les effectifs de population totale du registre manitobain sur la santé de la population de $2014^{30}$ et nous avons géocodé les données sur la population au niveau de l'îlot de diffusion à l'aide des codes postaux à six caractères.

TABLEAU 2

Classement du revenu par quintile, Région sanitaire de Winnipeg (Manitoba), Canada

\begin{tabular}{ccc} 
Quintile & $\begin{array}{c}\text { Revenu moyen du ménage dans l'aire de } \\
\text { diffusion où les revenus sont les plus } \\
\text { faibles, pour chaque quintile }(\$)\end{array}$ & $\begin{array}{c}\text { Revenu moyen du ménage dans l'aire de } \\
\text { diffusion où les revenus sont les plus } \\
\text { élevés, pour chaque quintile }(\$)\end{array}$ \\
\hline 1 & 14772 & 49506 \\
2 & 49509 & 63475 \\
3 & 63513 & 78890 \\
4 & 78957 & 98953 \\
5 & 98963 & 343154 \\
\hline
\end{tabular}

Source des données : Statistique Canada, Recensement de la population de 2011.
Nous avons en quatrième lieu calculé la distance géodésique (la plus courte distance "à vol d'oiseau ») entre le centroïde de chacun des 5500 îlots de diffusion et chaque épicerie appartenant à une chaîne nationale et chaque épicerie à service complet la plus proche afin de compléter nos deux scénarios sur les déserts alimentaires. Nous avons choisi cette méthode car elle permet d'améliorer la reproductibilité sans trop influencer les résultats. Nous avons convenu qu'une distance de 500 mètres ou moins était raisonnable pour se rendre à pied à l'épicerie, distance aussi utilisée par d'autres chercheurs ${ }^{4,31}$ : comme il fait très froid à Winnipeg, la neige recouvrant le sol pendant plus du tiers de l'année (de novembre au début du mois d'avril), 500 mètres semble une distance raisonnable quand il faut marcher en portant des provisions, parfois avec des enfants.

Enfin, nous avons évalué les îlots de diffusion comme des déserts alimentaires en suivant les deux scénarios, décrits dans le tableau 1. Nous avons ensuite calculé la population totale vivant dans un désert alimentaire de la Région sanitaire de Winnipeg en additionnant les effectifs de population des îlots de diffusion classés comme déserts alimentaires. Tous les calculs ont été effectués à l'aide de la version 3.5.4 du logiciel Epi Info ${ }^{32}$ au moyen de scripts automatisés, et les données des tableaux ont été exportées dans le logiciel ArcGIS $10.2^{33}$ pour en établir la cartographie. 


\section{Résultats}

La figure 1 présente la distribution, par quintile de revenu, des îlots de diffusion de la Région sanitaire de Winnipeg. La plupart des quartiers à faible revenu se trouvent dans le centre-ville de Winnipeg, mais certains sont localisés dans des banlieues éloignées.

La figure 2 présente l'emplacement des déserts alimentaires dans la Région sanitaire de Winnipeg d'après le scénario 1 , qui a été établi en fonction de la distance entre un quartier à faible revenu et la plus proche épicerie appartenant à une chaîne nationale. Selon cette méthode, un nombre important d'îlots de diffusion ont été classés comme déserts alimentaires dans le centre-ville de Winnipeg et un plus petit nombre, dispersé dans les banlieues, a répondu également à ces critères de définition. Avec le scénario 1, 104335 personnes (14,49\% de la population) de la Région sanitaire de Winnipeg vivaient dans un désert alimentaire en 2014.

La figure 3 présente l'emplacement des déserts alimentaires d'après le scénario 2, qui a été établi en fonction de la distance entre un quartier à faible revenu et une épicerie appartenant à une chaîne nationale ou une épicerie à service complet. Le fait d'inclure les épiceries à service complet dans l'analyse conduit à un nombre considérablement plus petit d'îlots de diffusion classés comme déserts alimentaires dans le centre-ville comparativement au scénario 1 . Par contre, la plupart des déserts alimentaires situés en banlieue repérés dans le scénario 1 ont également été qualifiés de déserts alimentaires avec le scénario 2 . Avec le scénario 2, 64574 personnes $(9,1 \%$ de la population) de la Région sanitaire de Winnipeg vivaient dans un désert alimentaire en 2014.

\section{Analyse}

Les résultats de cette étude indiquent qu'il existe des déserts alimentaires dans la Région sanitaire de Winnipeg et que cela touche une proportion importante de la population. Dans le cadre de notre étude, deux scénarios sur les déserts alimentaires ont été mis au point pour les résidents des quartiers à faible revenu : un scénario qui tient compte uniquement de la proximité des épiceries appartenant à une chaîne nationale, et un scénario qui tient compte de la proximité des épiceries appartenant à
FIGURE 1

Revenu moyen du ménage selon le quintile de revenu, Région sanitaire de Winnipeg, 2011

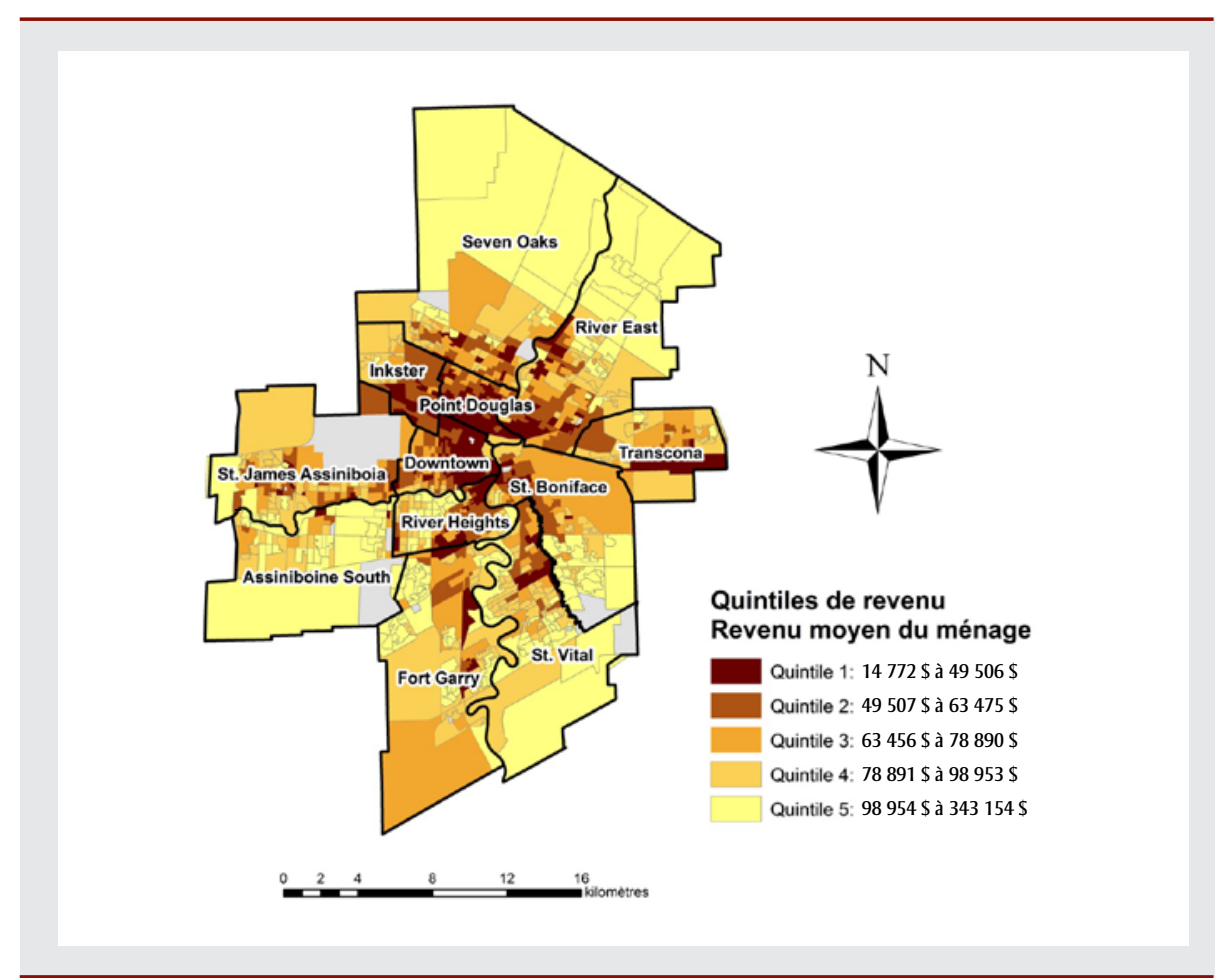

Source des données : Statistique Canada, Recensement de la population de 2011.

FIGURE 2

Scénario 1 sur les déserts alimentaires : déserts alimentaires dans la Région sanitaire de Winnipeg en fonction uniquement de la proximité d'une épicerie appartenant à une chaîne nationale

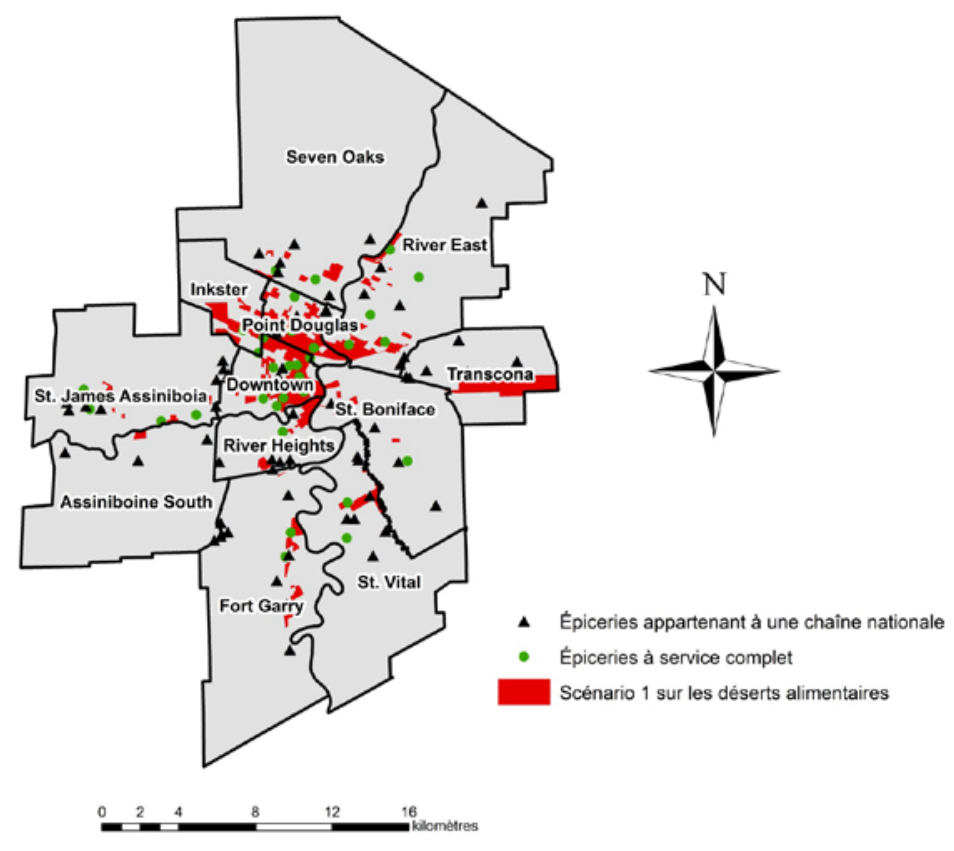


FIGURE 3

Scénario 2 sur les déserts alimentaires : déserts alimentaires dans la Région sanitaire de Winnipeg, en fonction de la proximité d'une épicerie appartenant à une chaîne nationale ou d'une épicerie à service complet

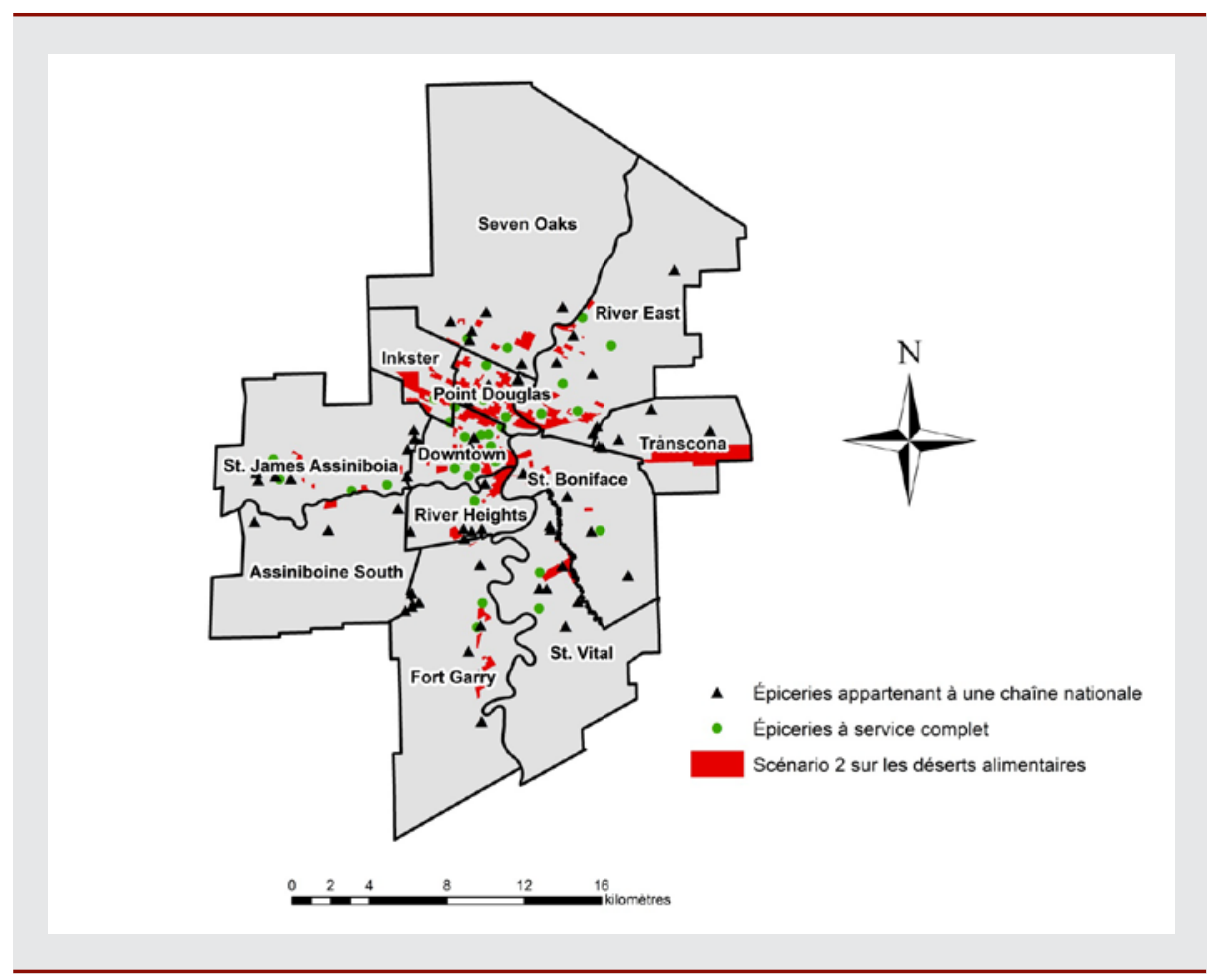

une chaîne nationale et des épiceries à service complet. Dans le scénario du meilleur cas, soit le second scénario susmentionné, on estime que $9 \%$ des résidents de Winnipeg (près d'une personne sur dix) vivent dans un désert alimentaire. Si l'on considère uniquement la proximité d'une épicerie appartenant à une chaîne nationale, près de $15 \%$ des résidents de Winnipeg (une personne sur huit, ou 104335 personnes) vivent dans un désert alimentaire. Nos cartes montrent que les déserts alimentaires se concentrent dans le centre-ville de Winnipeg, mais qu'il en existe également en banlieue. Ces résultats concordent avec les résultats observés dans certaines autres villes canadiennes, où l'on a repéré un nombre important de résidents à faible revenu ayant difficilement accès à des aliments sains ${ }^{10,20,21,23}$.

Winnipeg compte l'une des plus importantes populations urbaines pauvres au Canada : en 2010, 17,5 \% des Winnipégois vivaient en situation de faible revenu, contre $9 \%$ de l'ensemble de la population ${ }^{34}$. Plus précisément, les quartiers résidentiels du centre-ville de Winnipeg, qui abritent le plus grand nombre de déserts alimentaires, soit Downtown et Point Douglas, correspondaient à des revenus médians du ménage respectifs de 36298 \$ et de 39614 \$ en 2010, contre 58503 \$ dans l'ensemble de la Région sanitaire de Winnipeg ${ }^{35}$. Un revenu faible réduit les options de transport, et il a été démontré qu'un accès inadéquat à des moyens de transport abordables constitue un obstacle à l'accès à une quantité suffisante d'aliments sains ${ }^{2,3}$. Les ménages à faible revenu ont un accès moindre à des véhicules personnels et conduisent moins que les ménages à revenu élevé ${ }^{36,37}$. Un accès difficile à des aliments abordables et nutritifs ainsi qu'un faible revenu constituent donc un double fardeau pour un grand nombre de Winnipégois qui vivent dans un désert alimentaire. Ces derniers doivent d'établissements pour se nourrir, comme les dépanneurs, les banques alimentaires et les restaurants-minute bon marché (p. ex. ceux qui proposent de la pizza à $1 \$$ ), soit sur les taxis (qui coûtent cher) et les réseaux personnels (qui peuvent être peu commodes et peu fiables) s'ils sont disponibles, pour se rendre dans les grandes épiceries et en revenir.

La persistance des déserts alimentaires dans les banlieues toujours présente lorsqu'on tenait aussi compte des épiceries à service complet montre que ces quartiers donc compter soit sur d'autres types sont principalement desservis par de grandes épiceries appartenant à une chaîne nationale, ce qui concorde avec les tendances en matière de commerce de détail en alimentation : les grandes épiceries appartenant à une chaîne prennent la place des petites chaînes ou des épiceries indépendantes. Bedore qualifie ce phénomène de [traduction] « industrie [de l'alimentation au détail] à grande échelle non intégrée au quartier qui prédomine actuellement dans le paysage alimentaire $»^{38}$.

\section{Forces et limites}

Notre étude apporte une contribution originale à la littérature sur l'alimentation en lien avec l'environnement bâti en ce qui a trait à la méthodologie. Premièrement, elle a permis de montrer qu'une méthode reproductible et relativement simple utilisant seulement trois types de données (emplacement des magasins d'alimentation, revenu du ménage et effectifs de population) pouvait servir à produire une analyse solide sur les déserts alimentaires pour un grand centre urbain. Cette approche pragmatique est particulièrement importante dans la mesure où les services de santé publique n'ont généralement pas les ressources pour effectuer régulièrement des analyses complexes sur l'environnement alimentaire. De plus, nous avons constaté que la simplicité relative de notre méthodologie a facilité la mobilisation des connaissances auprès des principaux intervenants communautaires, notamment les nutritionnistes communautaires, les militants de la cause alimentaire et les responsables des politiques.

Deuxièmement, notre étude a permis de montrer que tous les calculs servant à repérer les déserts alimentaires peuvent être effectués au moyen de scripts automatisés dans le logiciel Epi Info, et les tableaux sont faciles à importer dans le logiciel ArcGIS pour en produire la cartographie. Cette approche présente un avantage : l'analyse sur les déserts alimentaires peut être rapidement et facilement mise à jour en cas de besoin (p. ex. si un magasin d'alimentation ouvre ou ferme ses portes, ou si l'on souhaite analyser différents paramètres de proximité pour voir leurs effets). De telles situations se sont réellement produites pendant la période d'étude (plusieurs magasins d'alimentation ont fermé et deux magasins ont ouvert), et nous avons pu refaire l'analyse et produire de nouvelles cartes et estimations de la 
population avec un minimum d'efforts et de ressources.

Troisièmement, l'étude a démontré que le fait de tenir compte uniquement de la proximité des grands magasins d'alimentation appartenant à une chaîne nationale, comme c'est le cas d'autres analyses concernant les déserts alimentaires au Canada $^{21}$, peut entraîner une surestimation de la superficie des déserts alimentaires et de la population touchée. Dans de nombreux quartiers du centre-ville, les petites épiceries locales peuvent se révéler extrêmement importantes pour fournir un accès facile à un large éventail de produits alimentaires abordables. Comme nous l'avons démontré, le fait de prendre en compte la proximité des épiceries locales à service complet en plus de celle des épiceries appartenant à une chaîne nationale a réduit de $38 \%$ les estimations du nombre de personnes vivant dans un désert alimentaire dans la Région sanitaire de Winnipeg, soit de 104335 à 64574 .

Notre étude comporte plusieurs limites dont il faut tenir compte lors de l'interprétation des résultats. Premièrement, nous avons attribué aux ménages un revenu de façon globale, en fonction de leur résidence dans une aire de diffusion à faible revenu. Il est toutefois possible que certaines personnes à revenu élevé vivent dans des aires de diffusion à faible revenu et qu'elles ne soient pas aux prises avec des problèmes économiques ou de transport les empêchant d'avoir accès à une variété suffisante d'aliments sains. Par conséquent, nous avons pu surestimer la taille de la population vivant dans un désert alimentaire dans la Région sanitaire de Winnipeg. Cependant, cette surestimation pourrait être neutralisée par les personnes à faible revenu qui habitent dans des aires de diffusion à revenu élevé et qui n'ont donc pas été considérées comme des résidents de déserts alimentaires dans notre étude.

Deuxièmement, nous avons utilisé la distance géodésique (" à vol d'oiseau ») au lieu de la distance par le réseau routier (distance qu'une personne parcourt réellement en empruntant le réseau de rues) pour estimer la distance jusqu'au magasin d'alimentation le plus proche. Ce choix peut avoir nui à la précision de nos calculs de distance, mais l'erreur ainsi introduite a sans doute été minime dans les quartiers du centre-ville de Winnipeg, où la majorité des déserts alimentaires ont été repérés. En effet, dans le centre-ville de Winnipeg, la structure du réseau de rues est très dense (pâtés de maisons courts et nombreuses intersections), ce qui signifie que la distance géodésique est sans doute équivalente à la distance par le réseau routier, car les résidents peuvent emprunter de nombreux trajets directs pour se déplacer de leur logement à un magasin d'alimentation. Dans les quartiers de banlieue, la structure plus aérée des réseaux de rues a pu entraîner davantage de biais de classification.

Troisièmement, nous n'avons pas pris spécifiquement en compte les moyens de transport publics pour repérer les déserts alimentaires. Cependant, nous pensons que le revenu du ménage constitue un bon indice indirect de la capacité financière des personnes à se rendre facilement au magasin d'alimentation le plus proche, que ce soit en voiture, en taxi ou en autobus. À Winnipeg, le billet d'autobus est cher (particulièrement si un parent paie également pour les enfants qui l'accompagnent : par exemple, un billet pour adulte coûte 2,70 \$ et deux billets pour enfants coûtent 2,20\$ chacun, ce qui donne un total de 14,20 \$ pour l'aller-retour) et l'existence d'un trajet d'autobus local n'implique pas nécessairement un meilleur accès à un magasin d'alimentation éloigné (surtout pour un grand volume de produits d'épicerie). En outre, il arrive souvent que les autobus qui circulent des quartiers centraux de Winnipeg vers de grandes épiceries de banlieue soient peu pratiques et passent irrégulièrement.

Notre étude comporte une dernière limite : aucune mesure structurée (comme l'établissement officiel des coûts ou l'évaluation objective de la disponibilité des aliments en magasin) n'a été mise en place afin de classer les épiceries. Comme nous l'avons mentionné, les épiceries sélectionnées au départ l'ont été à l'aide des Pages Jaunes de la région, puis ont été vérifiées par des diététistes communautaires travaillant pour l'office régional de la santé local. L'évaluation structurée des caractéristiques de l'environnement des aliments vendus au détail (prix et variété) aurait été difficile et aurait nécessité beaucoup de ressources, ce qui explique peut-être pourquoi les autres études concernant les déserts alimentaires se sont concentrées uniquement sur les grandes épiceries appartenant à une chaîne nationale, qui sont faciles à repérer. À notre avis, si les ressources le permettent, les études ultérieures devraient évaluer de façon plus structurée la variété des produits et leurs prix dans les épiceries locales, en utilisant des critères quantifiables. Toutefois, nous considérons que la vérification effectuée dans notre étude par des diététistes en santé publique de la région est fiable, compte tenu de leur connaissance approfondie des collectivités locales et des ressources limitées dont nous disposions. Les études ultérieures devraient examiner les effets des déserts alimentaires sur les habitudes alimentaires et sur la santé, ainsi que sur l'expérience des résidents qui vivent dans un désert alimentaire.

\section{Conclusion}

Notre étude a démontré que les déserts alimentaires touchent près d'une personne sur dix à Winnipeg, une ville où les taux de pauvreté sont durablement élevés. Nous avons constaté que les secteurs dépourvus d'épiceries et à bas revenus étaient concentrés dans le " noyau » ou le centre de la ville, avec néanmoins des zones touchées dans les banlieues. La nouvelle méthodologie employée dans notre étude, transparente et reproductible, permet d'exercer une surveillance régulière et favorise un engagement significatif de la part des collectivités, des magasins de détail et des responsables des politiques. Le fait de tenir compte à la fois des épiceries appartenant à une chaîne nationale et des épiceries locales à service complet permet une évaluation plus réaliste des déserts alimentaires. Un revenu adéquat constitue une priorité pour lutter contre l'insécurité alimentaire, mais l'évaluation de la prévalence des déserts alimentaires et présentation accessible des données permettent de mobiliser les connaissances et d'intégrer d'autres données importantes sur la sociodémographie et sur le paysage alimentaire dans l'analyse. Nos données concernant les déserts alimentaires ont été spatialisées, ce qui permet l'ajout ultérieur de nouvelles couches de données pertinentes touchant les secteurs constituant des déserts alimentaires sur des cartes ainsi que dans des applications interactives de cartographie, comme Google Earth $^{39}$, fournissant ainsi un outil puissant pour encourager la participation des intervenants.

\section{Remerciements}

Les auteurs aimeraient remercier les diététistes en santé publique de l'Office régional de la santé de Winnipeg pour leur aide précieuse dans la réalisation de cette étude. 


\section{Conflits d'intérêts}

Les auteurs déclarent n'avoir aucun conflit d'intérêts.

\section{Contributions des auteurs}

Joyce Slater, Chris Green et Stefan EppKoop ont contribué à la conception de l'étude, à l'analyse des données et à la rédaction du manuscrit. Meghan Jakilazek a contribué à l'analyse des données. Tous les auteurs ont contribué à l'interprétation des résultats, ont revu le manuscrit et en ont approuvé la version définitive.

\section{Références}

1. Cummins S, Macintyre S. "Food deserts"-evidence and assumption in health policy making. BMJ. 2002; 325(7361):436-438.

2. Páez A, Mercado RG, Farber $S$, Morency C, Roorda M. Relative accessibility deprivation indicators for urban settings: definitions and application to food deserts in Montréal. Urban Stud. 2010;47(7):1415-1438.

3. Tsang S, Holt AM, Azevedo E. Évaluation des obstacles empêchant l'accès aux denrées chez les habitants de Cobourg (Ontario) en situation d'insécurité alimentaire. Maladies chroniques et blessures au Canada. 2011; 31(3):133-140.

4. Wrigley N. "Food deserts" in British cities: policy context and research priorities. Urban Stud. 2002;39(11):20292040. En ligne à : http://usj.sagepub. com/content/39/11/2029.full.pdf

5. Moore L V, Diez Roux A V, Nettleton JA, Jacobs DR. Associations of the local food environment with diet quality-a comparison of assessments based on surveys and geographic information systems: the multi-ethnic study of atherosclerosis. Am J Epidemiol. 2008;167(8):917-924.

6. Chen D, Jaenicke EC, Volpe RJ. Food environments and obesity: household diet expenditure versus food deserts. Am J Public Health. 2016;106(5): 881-888.

7. Shaw HJ. Food deserts: Towards the development of a classification. Geogr Ann Ser B Hum Geogr. 2006;88(2): 231-247.
8. Walker RE, Keane CR, Burke JG Disparities and access to healthy food in the United States: a review of food deserts literature. Health and Place. 2010;16(5):876-884.

9. McEntee J. Highlighting food inadequacies: does the food desert metaphor help this cause? Br Food J. 2009; 111(4):349-363.

10. Wiebe, K, Distasio J, Shirtliffe, R. Confronting the illusion: developing a method to identify food mirages and food deserts in Winnipeg [Internet]. Winnipeg (MB): The University of Manitoba; 2016. En ligne à : http:// winnspace.uwinnipeg.ca/handle /10680/1205

11. Breyer B, Voss-Andreae A. Food mirages: geographic and economic barriers to healthful food access in Portland, Oregon. Health and Place. 2013;24:131-139.

12. Coveney J, O’Dwyer LA. Effects of mobility and location on food access. Health and Place. 2009;15(1):45-55.

13. Macdonald L, Ellaway A, Ball K, Macintyre S. Is proximity to a food retail store associated with diet and BMI in Glasgow, Scotland? BMC Public Health. 2011;11(1):464. En ligne à : http://dx.doi.org/10.1186/1471 $-2458-11-464$

14. Alviola PA, Nayga RM, Thomsen MR, Wang Z. Determinants of food deserts. Am J Agric Econ. 2013;95(5):1259-1265.

15. Polsky JY, Moineddin R, Glazier RH, Dunn JR, Booth GL. Foodscapes of southern Ontario: neighbourhood deprivation and access to healthy and unhealthy food retail. Can J Public Health. 2014; 105(5):e369-e375.

16. Chen H-. J, Wang Y. The changing food outlet distributions and local contextual factors in the United States. BMC Public Health. 2014;14:42. doi : 10.1186/1471-2458-14-42.

17. Beaulac J, Kristjansson E, Cummins S. A systematic review of food deserts, 1966-2007. Prev Chronic Dis. 2009; 6(3):A105. En ligne à : https://www .ncbi.nlm.nih.gov/pmc/articles /PMC2722409/
18. Whelan A, Wrigley N, Warm D, Cannings E. Life in a "Food Desert." Urban Stud. 2002;39(11):2083-2100. En ligne à : http://journals.sagepub.com/ doi/abs/10.1080/0042098022000011371

19. Wrigley N, Warm D, Margetts B, Whelan A. Assessing the Impact of Improved Retail Access on Diet in a "Food Desert": A Preliminary Report. Urban Stud. 2002;39(11):2061-2082.

20. Larsen K, Gilliland J. Mapping the evolution of "food deserts" in a Canadian city: supermarket accessibility in London, Ontario, 1961-2005. Int J Health Geogr. 2008;7(1):16. En ligne à : http://www.ij-healthgeographics .com/content/7/1/16

21. Gould AC, Apparicio P, Cloutier MS. Classifying neighbourhoods by level of access to stores selling fresh fruit and vegetables and groceries: identifying problematic areas in the city of Gatineau, Quebec. Can J Public Health. 2012;103(6):e433-e437.

22. Smoyer-Tomic KE, Spence JC, Amrhein C. Food deserts in the prairies? Supermarket accessibility and neighborhood need in Edmonton, Canada. Prof Geogr. 2006;58(3):307-326.

23. Cushon J, Creighton $\mathrm{T}$, Kershaw $\mathrm{T}$, Marko J, Markham T. Défavorisation, accès aux aliments et équilibre alimentaire à Saskatoon (Saskatchewan). Maladies chroniques et blessures au Canada. 2013;33(3):165-178.

24. Luan H, Law J, Quick M. Identifying food deserts and swamps based on relative healthy food access: a spatiotemporal Bayesian approach. Int $\mathrm{J}$ Health Geogr [Internet]. 2015;14(1):37. doi : 10.1186/s12942-015-0030-8.

25. Minaker LM, Shuh A, Olstad DL, Engler-Stringer R, Black JL, Mah CL. Retail food environments research in Canada: a scoping review. Can J Public Health. 2016;107:5344. doi : 10.17269/cjph.107.5344.

26. Apparicio P, Cloutier MS, Shearmur R. The case of Montréal's missing food deserts: evaluation of accessibility to food supermarkets. Int J Health Geogr. 2007;12;6:4. doi : 10.1186/1476-072X $-6-4$. 
27. Bertrand L, Thérien F, Cloutier MS. Measuring and mapping disparities in access to fresh fruits and vegetables in Montréal. Can J Public Health. 2008; 99(1):6-11.

28. Nicholson, Katie; Marcoux J. Buying groceries a long trek for family in Winnipeg food desert [Internet]. CBC News. 2015 (2 déc.). En ligne à : http://www.cbc.ca/news/canada /manitoba/buying-groceries-a-long -trek-for-family-in-winnipeg-food -desert-1.3345126

29. Manitoba Centre for Health Policy. Concept: income quintile ranking procedure [Internet]. Winnipeg (MB): Manitoba Centre for Health Policy. 2002. En ligne à : http://mchp-appserv .cpe.umanitoba.ca/viewConcept.php ?conceptID $=1164$

30. Rady Faculty of Health Sciences. Manitoba population health research data repository data list [Internet]. Rady Faculty of Health Sciences. 2017. En ligne à : http://umanitoba.ca /faculties/health_sciences/medicine /units/chs/departmental_units/mchp /resources/repository/datalist.html

31. Furey S, Strugnell C, Mcilveen H. An investigation of the potential existence of "food deserts" in rural and urban areas of Northern Ireland. Agric Human Values. 2001;18(4):447-457.

32. Epi Info version 3.5.4 (Centers for Disease Control and Prevention, Atlanta, GA, USA); 2012. En ligne à : https:// www.cdc.gov/epiinfo/index.html

33. ArcGIS version 10.2 (Environmental Systems Research Institute [ESRI], Redlands, CA, USA); 2012.

34. Statistique Canada. Personnes ayant un faible revenu après impôt [Internet]. Ottawa (Ont.) : Statistique Canada; 2012 [modification le 27 juin 2013]. En ligne à : http://www.statcan .gc.ca/tables-tableaux/sum-som/102 /cst01/famil19a-fra.htm

35. City of Winnipeg. Census information: 2011 census-City of Winnipeg neighbourhood profiles [Internet]. Winnipeg (MB): City of Winnipeg; 2013 [modification le 11 octobre 2016]. En ligne à : http://winnipeg.ca/census/2011
36. Berube A, Deakin E, Raphael S Socioeconomic differences in household automobile ownership rates: implications for evacuation policy [Internet]. Washington (DC): The Brookings Institution; 2006. En ligne à : http://socrates.berkeley.edu/ raphael /BerubeDeakenRaphael.pdf

37. Statistique Canada. Enquête sur les dépenses des ménages, 2013 [Internet]. Ottawa (Ont.) : Statistique Canada; 2013 [modification le 21 décembre 2015]. En ligne à : http://www.statcan .gc.ca/daily-quotidien/150122 /dq150122b-cansim-fra.htm

38. Bedore M. Geographies of capital formation and rescaling: a historical-geographical approach to the food desert problem. Can Geogr. 2013;57(2):133-153.

39. Google Earth [Internet]. Mountain View (CA), Google, Inc.; 2016. En ligne à : https://www.google.ca/intl/fr/earth/ 